$11-1-2015$

\title{
Migrated Avalon Veno-Venous Extracorporeal Membrane Oxygenation Cannula: How to Adjust Without Interruption of Flow.
}

\author{
Daizo Tanaka \\ Thomas Jefferson University \\ Harrsion T. Pitcher \\ Thomas Jefferson University \\ Nicholas C. Cavarocchi \\ Thomas Jefferson University \\ Hitoshi Hirose \\ Thomas Jefferson University
}

Follow this and additional works at: https://jdc.jefferson.edu/surgeryfp

Part of the Surgery Commons

Let us know how access to this document benefits you

\section{Recommended Citation}

Tanaka, Daizo; Pitcher, Harrsion T.; Cavarocchi, Nicholas C.; and Hirose, Hitoshi, "Migrated Avalon Veno-Venous Extracorporeal Membrane Oxygenation Cannula: How to Adjust Without Interruption of Flow." (2015). Department of Surgery Faculty Papers. Paper 141.

https://jdc.jefferson.edu/surgeryfp/141

This Article is brought to you for free and open access by the Jefferson Digital Commons. The Jefferson Digital Commons is a service of Thomas Jefferson University's Center for Teaching and Learning (CTL). The Commons is a showcase for Jefferson books and journals, peer-reviewed scholarly publications, unique historical collections from the University archives, and teaching tools. The Jefferson Digital Commons allows researchers and interested readers anywhere in the world to learn about and keep up to date with Jefferson scholarship. This article has been accepted for inclusion in Department of Surgery Faculty Papers by an authorized administrator of the Jefferson Digital Commons. For more information, please contact: JeffersonDigitalCommons@jefferson.edu. 
Veno-venous extracorporeal membrane oxygenation (VV ECMO): Indications, Preprocedural considerations and Technique.

Aisha Shaheen MD, MHA, Daizo Tanaka, MD, Nicholas C. Cavarocchi MD, Hitoshi Hirose $\mathrm{MD}, \mathrm{Ph}$. D.

From Department of Surgery, Thomas Jefferson University, Philadelphia, PA

Short running title: VV ECMO cannulation technique.

Key words: technique ECMO, respiratory failure, ARDS, technique.

Corresponding Author:

Hitoshi Hirose, MD, PhD.

Division of Cardiothoracic Surgery, Department of Surgery, Thomas Jefferson University 607 College Building, 1025 Walnut Street, Philadelphia, PA 19107

Phone: 215-955-6996, Email: genex@nifty.com

There was no financial support for this project.

Abstract word count: 32

Main text word count: 2914 without references, 3416 with references

Number of table: 0

Number of figure: 3 


\begin{abstract}
Veno-venous extracorporeal membrane oxygenation (VV ECMO) has been used for refractory respiratory failure. We describe the indications, technical aspects, and outcomes of placing VV ECMO in adults using a dual-lumen, single-cannula catheter.
\end{abstract}




\section{Introduction}

Veno-venous extracorporeal membrane oxygenation (VV ECMO) is used for refractory respiratory failure. Previously, we described our technique of percutaneous placement of venoarterial (VA) ECMO cannulas. ${ }^{1}$ We now describe the percutaneous placement of a cannula for VV ECMO support, including indications, patient assessment, preparation for cannulation and the technical aspects of the procedure using a double lumen bi-caval cannula (Avalon, Maquet, Rastatt, Germany).

\section{Indications}

VV ECMO is reserved for patients with compromise of their ventilation or oxygenation status that is refractory to optimum mechanical ventilation and medical therapy. ${ }^{2-4}$ In VV ECMO, deoxygenated blood is drained from the venous system, and sent through an external circuit which contains a gas-permeable membrane that removes carbon dioxide and an oxygenator that oxygenates the blood. Thereafter the blood is returned to the venous system, whereas in VA ECMO the oxygenated blood is returned to the arterial system. ${ }^{1}$ Distribution of the oxygenated blood to the patient in VV ECMO requires viability of the patient's own circulatory system and therefore VV ECMO depends on the patient having normal cardiac function to maintain adequate perfusion. ${ }^{4}$ Thus, the primary indication of VV ECMO is severe acute ventilatory and/or oxygenation failure that the patient is unlikely to survive with conventional mechanical ventilation but is potentially reversible and is not associated with cardiac failure. $^{2-4}$

Common conditions of reversible respiratory failure for which VV ECMO is a potential treatment option include adult respiratory distress syndrome (ARDS) due to aspiration, viral/bacterial/atypical pneumonia, barotrauma, and acute on chronic interstitial pneumonitis. ${ }^{4}$ 
The patients who are not candidates for VV ECMO are those patients who have advanced and/or irreversible disease processes, such as overwhelming sepsis, non-pulmonary multi-organ failure, irreversible neurological injury, terminal illness or other life limiting disease. Additionally, patients with ventilator dependent respiratory failure or chronic respiratory failure that lack the potential to be bridged to lung transplantation are not candidates for VV ECMO. Finally, patients with cardiac dysfunction should receive VA ECMO support.

\section{Pre-procedural assessment}

Typical candidates are hypercapnic, hypoxic and unresponsive to optimal medical management, including low-tidal volumes, bronchodilator treatment, administration of epoprostenol, paralysis, and appropriate diuresis. ${ }^{2}$ It is important to establish the acute nature of the respiratory failure, ruling out cardiac or other non-pulmonary organ failure, and confirming a failure to improve with ventilator management. If it appears VV ECMO is clinically indicated and the patient would benefit from the therapy, the next step is to identify any patient factors that may prevent placement of the VV ECMO cannula.

The right internal jugular vein should be evaluated with ultrasound to identify intrinsic or acquired vascular pathologies including deep vein thrombosis, stenosis or previous stent placements that may preclude cannula placement. The placement of cannulas may be technically difficult in morbidly obese patients. Additional information such as coagulopathies or any contraindications to anticoagulation should be noted. Echocardiography should be performed before the procedure to rule out cardiac dysfunction or structural abnormalities such as a patent foramen ovale or tricuspid regurgitation. ${ }^{5}$ The presence of those structural abnormalities may cause inadequate mixing of ECMO inflow and outflow. Additionally, VV ECMO placement in a 
patient with cardiac failure of either side can result in cardiac over-distension and worsening pulmonary edema.

The protocol at our institution for patients who meet criteria for VV ECMO but who are not candidates for this system secondary to limitations of venous access, cardiac dysfunction or a structural abnormality is to place VA ECMO.

The local internal review board approved this presentation.

\section{Traditional VV ECMO cannulation}

Prior to the advent of the Avalon cannula, placement of VV ECMO support required multiple cannulation sites. The traditional VV ECMO system involved bilateral groin cannulations with one long venous cannula extending into the right atrium serving as inflow of oxygenated blood to the patient and one short venous cannula ending in the inferior vena cava (IVC) serving as outflow of the unoxygenated blood (Figure 1A). A second variation of the preAvalon VV ECMO system consisted of an outflow cannula in the groin ending in the IVC and a inflow cannula in the right atrium placed from the upper extremity (Figure 1B). These VV ECMO systems may result in ineffective oxygenation via ECMO due to the proximity of the inflow and outflow cannulas: the outflow cannula could be drawn back into the ECMO circuit without going into the patient's circulatory system. Another potential issue of these double cannulation systems was the potential for low ECMO flow related to a relatively easily collapsible IVC due to suction effect from the outflow cannula, which often observed in low intravascular volume status.

\section{Avalon double lumen bi-caval cannula}

In our practice, we use a double lumen bi-caval cannula (Avalon cannula) for VV ECMO. This Avalon cannula is a rigid straight cannula that was designed to be inserted from the 
right internal jugular vein into the IVC. Due to the rigidity of the cannula, other upper extremity cannulation such as subclavian or left internal jugular vein access is not recommended. The Avalon catheter has 3 ports referred to as the proximal, middle and distal ports. The distal port located at the tip of the Avalon cannula should be placed just below the atrial-IVC junction. Once the distal port is positioned inside the proximal portion of the IVC, the proximal port of the cannula should be in the SVC. These proximal and distal ports drain unoxygenated blood from the upper and lower body, respectively. The middle port of the Avalon catheter should rest in the right atrium and ideally face towards the tricuspid valve. Oxygenated blood from ECMO circuit is delivered to this middle port. Then the oxygenated blood is circulated to the pulmonary vasculature followed by the systemic circulation by native cardiac function. ${ }^{6,7}$ The Avalon cannula has specially designed to minimize inflow-outflow reperfusion and shunting of oxygenated blood.

The additional advantage of the dual-lumen design of the Avalon catheter is that it does not require femoral cannulation. Femoral cannulation hinders the ability to use prone positioning, which is a common secondary intervention performed in VV ECMO patients to improve oxygenation and lung recruitment. Avoiding a femoral cannulation also allows patients to sit in bed or even ambulate while on VV ECMO support. ${ }^{4}$

\section{Cannulation technique}

Prior to initiation of the procedure, a central venous line and an arterial line should be in place for proper monitoring of hemodynamics and access available for infusion of vasopressors and volume as needed throughout the procedure. If the right internal jugular vein is already used for central line access, an alternative central line should be obtained since the Avalon cannula is going to be placed in the right internal jugular vein. Echocardiography should be available at the 
bedside. If adequate visualization of the cardiac structures was not obtained by transthoracic echocardiography, transesophageal echocardiography should be used and the echo probe should be in position prior to skin prep for ECMO cannulation.

Percutaneous placement of VV ECMO cannulas can be performed in a variety of hospital settings including an emergency room, a cardiac cauterization laboratory, an intensive care unit or an operating room; however use of fluoroscopy is strongly recommended to ensure proper positioning of the wires and the cannula. We prefer to use a percutaneous approach to place the cannula as this minimizes bleeding from the cannulation site. The procedure is preferentially performed in the intensive care unit with fluoroscopy with trained personnel, support staff and the necessary equipment being readily available. Prior to the procedure, the patient needs to be sedated adequately with an airway properly secured.

Two operators should be available to control the wires and cannulas. The patient is prepped and draped sterilely and a single dose of intravenous antibiotics is administered. We routinely prep both groins for a possible conversion to VA ECMO in case we fail to obtain right internal jugular vein access.

Percutaneous puncture of the right internal jugular vein is performed under ultrasound guidance. The puncture site should be some distance from the clavicle. Otherwise, it is difficult to obtain adequate exposure upon decannulation or if the vessel needs to be repaired. Fluoroscopy is then used to pass a flexible j-tip guidewire from the internal jugular vein into the IVC. The tip of the guide wire should be placed beyond renal veins to avoid dislodgment or migration of the wire (Figure 2A). If the guidewire is not deep enough, the cannula may migrate to the right ventricle or to the hepatic vein. The Avalon insertion kit (Avalon elite vascular access kit, Maquet, Rastatt, Germany) includes one flexible j-tip 0.038 inch x $210 \mathrm{~cm}$ guidewire 
and serial dilators (10 Fr, 12 Fr, 16 Fr, 20 Fr and 24 Fr). The wire in the Avalon cannulation kit may be too flexible to manipulate the wire to the appropriate position. Occasionally, the presence of a large Eustachian valve at the caval-atrial junction may cause the wire to travel into the right ventricle instead of the IVC. In this case, a stiffer J-tip wire $(0.038$ inch $x 210 \mathrm{~cm}$, Edwards Lifescience LLC, Irvine, CA) or alternative stiffer wires including the Amplatz Stiff Wire $^{\text {TM }}$ or Amplatz Super Stiff Wire ${ }^{\text {TM }}$ (Boston Scientific, Washington, DC) may be substituted for the flexible wire in the Avalon kit. Using the Seldinger technique, the access site is progressively enlarged with serial dilators placed over the (Figure 2B). Thick skin or fascia may be present which requires forceful manipulation of the dilators until the access site is sizeable enough to accommodate placement of the cannula. During the vigorous dilatation of the soft tissue, the wire may kink or dislodge. Confirmation of the position of the wire is necessary with fluoroscopy each time the dilator is used. The access site should be held with a finger to minimize the blood loss whenever dilators are exchanged.

Cannulas are selected preoperatively based on manufacture pressure flow curves and the optimal flow calculated from a formula based on body surface area (BSA) x 2.2. We use this formula to provide optimal cardiac index. Usually, maximum flow is limited to $3 \mathrm{~L} / \mathrm{min}$ with use of a $23 \mathrm{Fr}$ cannula, $4.5 \mathrm{~L} / \mathrm{min}$ with a $27 \mathrm{Fr}$ cannula, and greater than $6.5 \mathrm{~L} / \mathrm{min}$ with $31 \mathrm{Fr}$ Avalon cannula. Although ECMO cannulas have the ability to run above the recommended flow, doing so may cause hemolysis; thus, in general, our policy is to utilize the largest cannula feasible.

Systemic anticoagulation is required to prevent forming thrombus in the ECMO circuit. Therefore, after the tract is dilated, a 5000 unit intravenous bolus of heparin (7500 units for BSA >2.2) is administered to prevent thrombosis prior to placement of the Avalon cannula. An inner stylet was placed in the Avalon cannula to facilitate the insertion over the guide wire along the 
dilated tract. The tip of the inner stylet should be followed by fluoroscopy to ensure not to kink the wire by the sharp tip of the inner stylet. The inner stylet is partially withdrawn as the cannula is inserted into the SVC to avoid injury to the right atrium by the inner stylet. Fluoroscopy should be used to confirm the cannula tip terminates in the IVC (Figure 2C, Figure 3A). The wire and inner stylet are then completely removed and the two ends of the catheter (venous and arterial) are clamped. The ends of the cannula are connected to the ECMO circuit taking care to avoid introducing air bubbles into the tubing. The connection and circuit should be carefully and continuously examined for trapped air prior to unclamping, given the potentially catastrophic complications that would result both from air emboli to the patient and the no-flow phenomenon due to disturbance of the centrifugal pump in the ECMO circuit. Once both the venous and arterial sides of the catheter are connected to the ECMO circuit, all clamps are removed and the circuit is started on full flow (approximately 4-5 L/min, BSA x $2.2 \mathrm{~L} / \mathrm{min}$ ).

At this point a bedside echocardiography is performed to confirm the middle port of the catheter is positioned in the right atrium with outflow jetting into the tricuspid valve (Figure 3B). ${ }^{5}$ The cannula can be moved manually to position the middle port of the cannula if needed. The catheter is secured to the skin with several sutures.

The entire procedure takes about 30 minutes with minimal blood loss (less than 100cc is expected). However, it is advisable to have two units of packed red blood cells as well as vasopressor support readily available. During the first a few hours of the ECMO, volume replacement is often necessary to keep adequate ECMO flow. The chattering of the ECMO tubing may be the first indication of intravascular volume depletion. 
Initial ECMO settings are $100 \% \mathrm{FiO}_{2}$ and a sweep of 5 to $6 \mathrm{~L} / \mathrm{min}$. Once the patient is placed on ECMO, ventilator settings can be reduced to appropriate ARDSnet settings: tidal volume 4-6 cc/kg, ventilator rate of 10 breaths /min, and a PEEP of 8-10 $\mathrm{cm} \mathrm{H}_{2} \mathrm{O}$ to prevent barotrauma. An arterial blood gas should be drawn 20 minutes after initiation of ECMO. Based on the arterial blood gas, the ECMO sweep is adjusted to allow for adequate $\mathrm{CO}_{2}$ clearance. Titration of respiratory rate or tidal volume settings on the ventilator is usually not necessary since the ECMO can be adjusted to clear $\mathrm{CO}_{2}$. To minimize oxygen toxicity, the $\mathrm{FiO}_{2}$ of the ventilator should be reduced as tolerated followed by reduction of the $\mathrm{FiO}_{2}$ of the ECMO. The oxygenation goal of VV ECMO is a $\mathrm{FiO}_{2}$ of $50 \%$ from both the ventilator and the VV ECMO circuit.

Once initial stabilization is achieved and the patient demonstrates improved oxygenation, secondary measures to facilitate alveolar improvement and overall oxygenation can be implemented. Bronchoscopy should be performed for pulmonary toilet and sampling of bronchial secretions for culture. The prone positioning bed (Rotaprone bed, KCI, San Antonio, TX) can be utilized to facilitate recruitment of the lung. In our practice, the patients on VV ECMO are deeply sedated to avoid the risk of cannula migration, to minimize bleeding from the cannula, and to allow for use of the prone positioning bed until recovery of the lung injury. In the younger patient population, sedatives such as ketamine may be necessary in addition to midazolam and fentanyl. These patients with acute respiratory failure could be different from the those who with chronic respiratory failure or patients who are candidates for lung transplantation. can potentially be extubated and ambulate while on the ECMO. In clinical situations involving persistent ARDS, once bacterial infection is excluded, we administer highdose corticosteroids per the Meduri protocol. ${ }^{4}$ 
Anticoagulation using a continuous heparin infusion is started 24 hours after placement of the ECMO circuit and monitored using the prothrombin time (PTT), with a therapeutic goal of 45-55 sec. In instances of bleeding from the cannulation or other sites, the anticoagulation can be held for up to 48 hours.

The patient is continuously evaluated for weaning from the VV ECMO system based on respiratory function parameters including compliance and the daily chest $\mathrm{x}$-ray findings. Once a $\mathrm{FiO}_{2}$ requirement of less than $50 \%$ is achieved on the ECMO circuit and the ventilator and the chest X-ray demonstrates improvement, tidal volume and respiratory setting is gradually increased on the ventilator and the sweep of the ECMO is reduced. Once a minimum sweep of less than $2 \mathrm{~L} / \mathrm{min}$ is achieved, the ECMO can be removed. In instances where airway pressure rises with ventilator manipulation, the patient is not considered stable enough for decannulation and ECMO support should be continued until pulmonary function fully recovers. Open decannulation and primary repair of the vessel is recommended for decannulation of ECMO, as complications including unexpected bleeding are more readily addressed.

\section{Our experience of VV ECMO}

Institutional review board approval was obtained to present our 5-year institutional experience. Between July 2010 and June 2015, a total of 45 VV ECMOs were performed for acute respiratory failure with severe ARDS secondary to viral pneumonia (16), bacterial, fungal or PCP pneumonia (6) aspiration (10), interstitial pneumonitis (5), pancreatitis related hypoxia (3), postcardiotomy respiratory failure (3), severe asthma (1), and others (1).

All patients were placed on VV ECMO with Avalon cannula. Early on, we experienced one incidence of cannula malposition into the right ventricle, which required surgical exploration. ${ }^{8}$ Now with our routine use of fluoroscopy for cannulation, we have not experienced 
any additional incidences of malposition of the cannula. However, there have been 5 incidences of cannula migrations, which required reposition of the cannula under either fluoroscopy or ultrasound. ${ }^{9}$ No patient required conversion to the VA ECMO or extra-venous cannula in addition to the Avalon cannula system.

The average length of VV ECMO was $11.5 \pm 6.7$ days. Thirty four patients $(76 \%)$ were successfully weaned from VV ECMO and survived of ECMO. The details of 11 patients who died during VV ECMO were sepsis secondary to pre-existing infection (3), cardiac death related to implantable cardiac assist device (2), stroke (2), non-recoverable pulmonary fibrosis (2), and ischemic bowel (2). After ECMO decannulation, 29 patients (64\%) were discharged home with appropriate neurological status.

\section{Conclusion}

Current practice of VV ECMO using a dual-lumen Avalon catheter requires experienced staff and adjunct equipment including ultrasound, echocardiography and fluoroscopy. Utilization of a standard protocol as described above will reduce complications and increase the likelihood of successful placement of VV ECMO. 


\section{References}

1. Lamb KM, Hirose H, Cavarocchi NC: Preparing and technical considerations for percutaneous cannulation for veno-arterial extracorporeal membrane oxygenation. J Card Surg 2013;28:190-192.

2. Brodie $\mathrm{D}$, Bacchetta M: Extracorporeal membrane oxygenation for ARDS in adults. N Eng J Med 2011;365:1905-1914.

3. Hosmane SR, Barrow T, Ashworth A, et al. Extracorporeal membrane oxygenation: a :radiologists' guide to who, what and where. Clinical Radiology 2015;70:e58-e66.

4. Hemmila M, Rowe S, Boules T, et al: Extracorporeal life support for severe acute respiratory distress syndrome in adults. Annals of Surgery 2004;240:595-607.

5. Platts DG, Sedgwick JF, Burstow DJ, et al: The role of echocardiography in the management of patient supported by extracorporeal membrane oxygenation. J Am Soc Echocardiogr 2012;25:131-141.

6. Bermudez CA, Rocha RV, Sappington PL, et al: Initial experience with single cannulation for venovenous extracorporeal oxygenation in adults. Ann Thorac Surg 2010;90:991-995. 7. Javidfar J, Brodie D, Wang D: Use of bicaval dual-lumen catheter for adult venovenous extracorporeal membrane oxygenation. An Thorac Surg 2011;91:1763-1768.

8. Hirose H, Yamane K, Marhefka G, et al: Right ventricular rupture and tamponade caused by malposition of the Avalon cannula for venovenous extracorporeal membrane oxygenation. $\mathrm{J}$ Cardiothorac Surg 2012; 7: 36.

9. Tanaka D, Pitcher HT, Cavarocchi N, et al: Migrated Avalon veno-venous extracorporeal membrane oxygenation cannula: How to adjust without interruption of flow. J Card Surg 2015;30:865-868. 


\section{Figure Legend}

Figure 1: Traditional VV ECMO circuit. Double groin access, one cannula in the right atrium for inflow to the patient and another cannula in the inferior vena cava for outflow from the patient (Figure 1A). Single groin access and upper extremity vein access, groin access for outflow and upper extremity for inflow (Figure 1B). The issue of this traditional VV ECMO is recirculation and shunting of the blood from ECMO due to the proximity of the cannulas and possible low flow due to collapse of the vein used for outflow from the patient.

Figure 2: Current Avalon cannulation technique. Guide wire placement to the inferior vena cava from right internal jugular vein (Figure 2A). Sequential dilatation of the access site is done (Figure 2B). The Avalon cannula is placed with distal port in the inferior vena cava and middle port facing to the tricuspid valve (Figure 2C).

Figure 3: Example of Avalon cannula position on chest x-ray (Figure 3A). Echocardiography shows good flow toward the tricuspid valve (Figure 3B). 